\title{
Central Serous Chorioretinopathy and Pregnancy: A Case Report
}

\author{
A. N. S. Kra, F. X. Kouassi, C. E. Koman, M. Soumahoro, T. Y. C. Sowagnon, Y. G. Ouffoue \\ Ophthalmology Department of the University Hospital Center of Cocody, Abidjan, Ivory Coast \\ Email: simeonalla@yahoo.com
}

How to cite this paper: Kra, A.N.S., Kouassi, F.X., Koman, C.E., Soumahoro, M., Sowagnon, T.Y.C. and Ouffoue, Y.G. (2018) Central Serous Chorioretinopathy and Pregnancy: A Case Report. Open Journal of Ophthalmology, 8, 207-213.

https://doi.org/10.4236/ojoph.2018.84024

Received: July 30, 2018

Accepted: October 12, 2018

Published: October 15, 2018

Copyright (c) 2018 by authors and Scientific Research Publishing Inc. This work is licensed under the Creative Commons Attribution International License (CC BY 4.0).

http://creativecommons.org/licenses/by/4.0/

\begin{abstract}
Introduction: Central serous chorioretinopathy (CSCR) is a macular retinal serous detachment associated with one or more leak points in the pigment epithelium. We present a case of spontaneous evolution in the $3^{\text {rd }}$ trimester of pregnancy in a primiparous woman. Observation: This was a 27 -year-old student, admitted for a sharp drop in visual acuity in the left eye. During the interrogation, a notion of 32 weeks of pregnancy-induced amenorrhea and a context of preparations for end-of-year school exams were noted. Ophthalmological examination revealed visual acuity in the left eye limited to counting the fingers at 3 meters. In the eye fundus, a bubble occupying the macular region of the left eye appeared. The examination of the right eye was strictly normal. The general examination was normal with normal blood pressure. Optical coherence tomography (OCT) brought out a large serous retinal detachment confirming the diagnosis. Faced with this diagnosis of central serous chorioretinopathy, therapeutic abstention with surveillance has been proposed. The delivery route was discussed because of the impact of pushing phases during vaginal delivery on the existing CSCR. Ophthalmologic examination performed 3 months after vaginal delivery was strictly normal with visual acuity at $10 / 10^{\text {th }}$ and total disappearance of retinal detachment at OCT. Discussion: Central serous chorioretinopathy is a relatively common ocular condition but whose pathophysiology is still controversial. Pregnancy is a known risk factor for central serous chorioretinopathy. The spontaneous resorption of central serous chorioretinopathy during pregnancy is reported in the literature, hence a therapeutic abstention with ophthalmological monitoring. Conclusion: Pregnancy in a primiparous woman is a stress factor that can promote central serous chorioretinitis.
\end{abstract}

\section{Keywords}

Central Serous Chorioretinopathy, Macula, Pregnancy, OCT 


\section{Introduction}

Central serous chorioretinopathy (CSCR) is a macular retinal serous detachment associated with one or more leak points in the pigment epithelium [1].

It is thought to occur as a result of a focal leakage at the level of the retinal pigment epithelium (RPE). The leakage manifests as an accumulation of extravasated fluid that accumulates in the subretinal space and separates the neurosensory retina from the RPE.

It is characterized by the presence of fluid under the neurosensory retina and affects mainly men (70\% of cases), young people (average age of 45 years). A number of risk factors have been reported to be associated with the condition including stress, steroids, pregnancy, Cushing's syndrome, hypertension, systemic lupus erythematosus, antibiotic and alcohol use, and allergic respiratory disease. In women it can be favored by pregnancy; which is a stress factor particularly in the primiparous woman.

The common presenting symptoms are blurred vision, central relative scotoma, micropsia, metamorphopsia, and color vision changes. In most cases, CSCR resolves within a few months, and vision subsequently returns to normal.

We report a case of favorable spontaneous evolution of central serous chorioretinopathy in the $3^{\text {rd }}$ trimester of pregnancy in a primiparous woman.

\section{Observation}

A 27-year-old female patient was admitted for a sharp drop in visual acuity in her left eye. During the interrogation, a pregnancy-induced amenorrhea of 32 weeks was noted in a primiparous woman during the end-of-year school exams.

The ophthalmological examination found a visual acuity in the left eye limited to counting the fingers at 3 meters.

In the eye fundus a raised retina was noted giving a bubble-like appearance occupying the macular region of the left eye without exudate or hemorrhage. The examination of the right eye was strictly normal.

The general examination was without peculiarities with good hemodynamic constants.

Optical coherence tomography (OCT) revealed a large serous retinal detachment with a thickness of 894 microns at the foveolar level (Figure 1).

Faced with this diagnosis of central serous chorioretinopathy, therapeutic abstention with surveillance was adopted. The delivery route was discussed, namely the impact of pushing phases during a vaginal delivery on the existing CSCR.

The patient finally gave birth vaginally without any particular incident.

Ophthalmologic examination performed 3 months after delivery was strictly normal with visual acuity at $10 / 10^{\text {th }}$ and at the OCT complete disappearance of serous retinal detachment was noted (Figure 2).

\section{Discussion}

Central serous chorioretinopathy (CSCR) is associated with macular retinal 

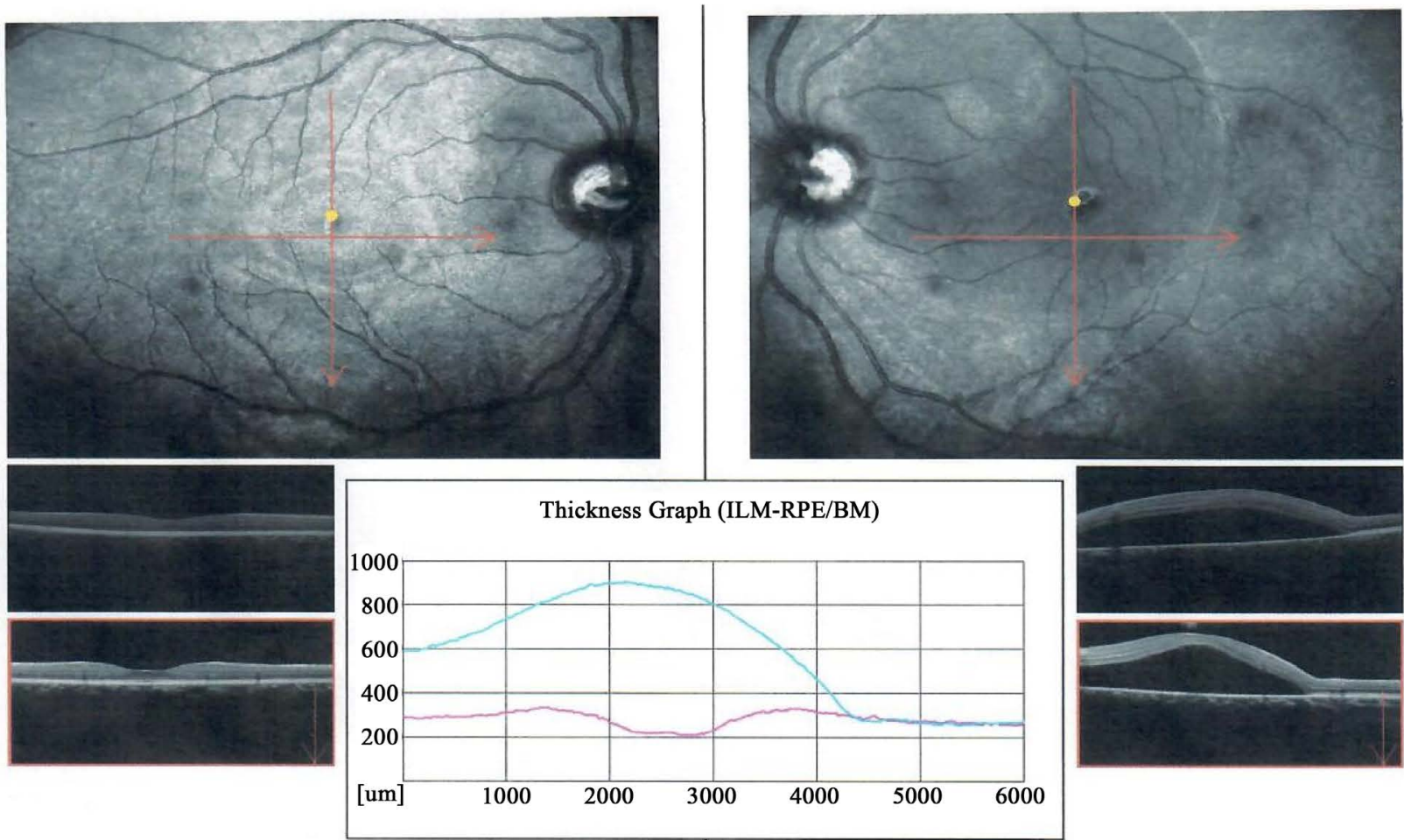
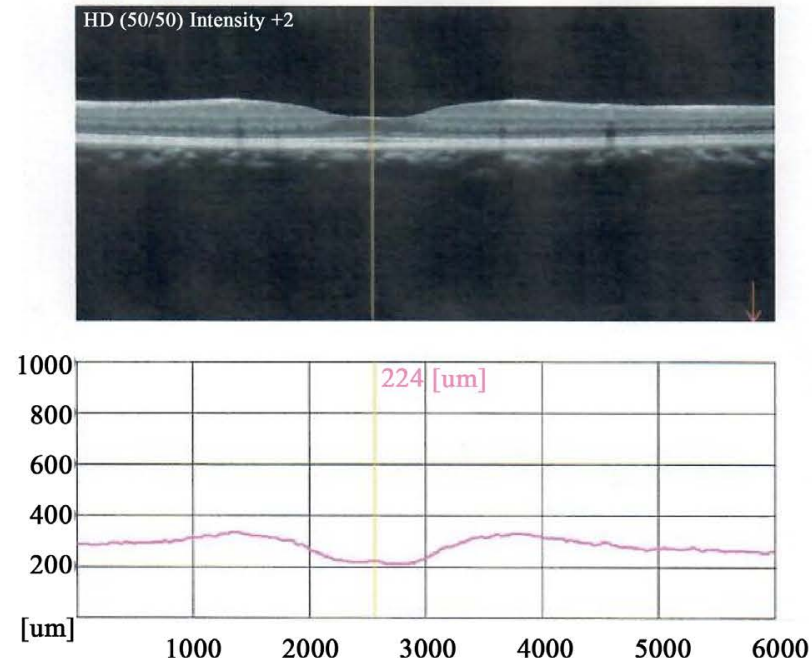
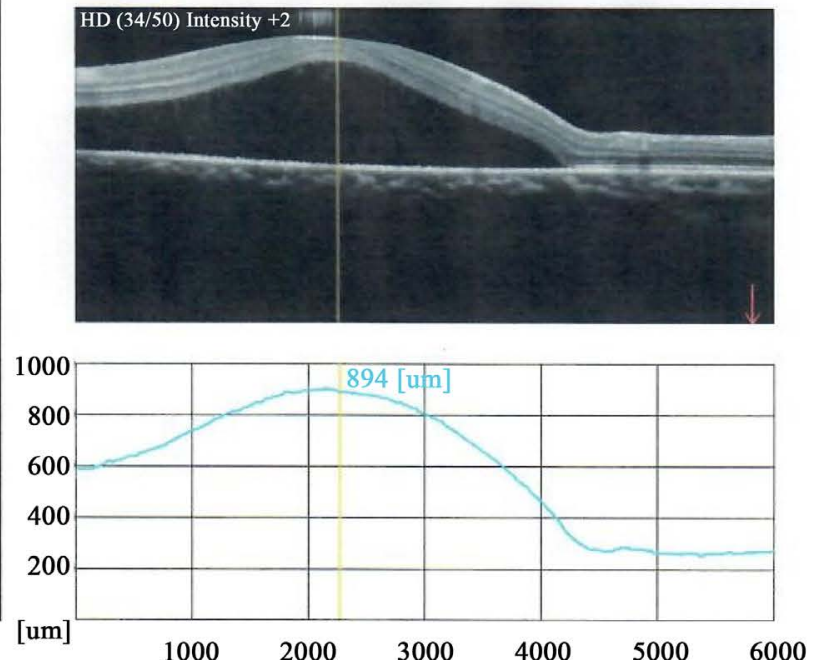

Figure 1. Optical coherence tomography (OCT) revealed a large serous retinal detachment with a thickness of 894 microns at the foveolar level.

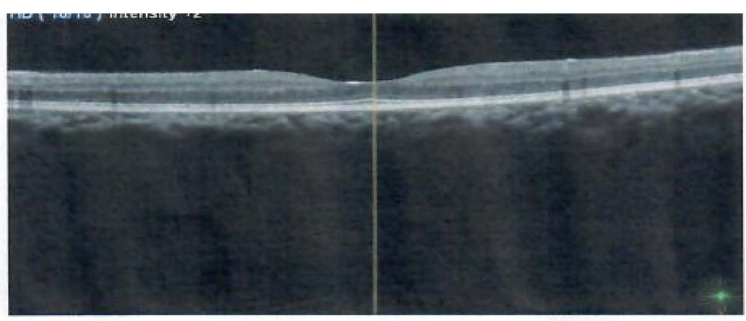

500

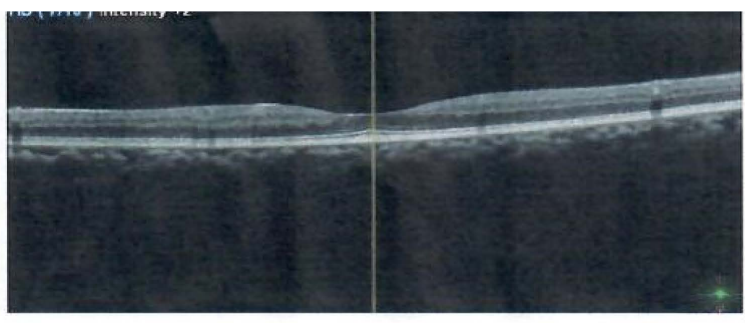

500

Figure 2. OCT complete disappearance of serous retinal detachment 3 month after delivery. 
serous detachment associated with one or more leak points in the pigment epithelium.

This disease is characterized by the accumulation of fluid under the central neurosensory retina, causing a more or less marked macular syndrome.

CSCR is a retinal pathology predominantly affecting young or middle-aged men. It would be favored by anxiety and is significantly aggravated by treatment with corticosteroids by general route or even local by skin ointments or nasal sprays [2].

In women it can be favored by pregnancy, especially in the primiparous woman with a context of intense stress.

The pathogenesis of CSCR in pregnancy is thought to be due to focal leak in the pigment epithelium and subsequent collection under macular neuroretinal membrane. This leak is thought to be related to abnormal choroidal circulation following capillary and venous congestion in the choroidal lobules responsible for ischemia and increased choroidal exudation because of hyper-permeability. There is excessive accumulation of choroidal fluid, which results in detachment of the EPR. The choroidal fluid passes through this opening in the sub-retinal neuronal space and produces detachment of the neurosensory retina [3].

CSCR is 6 - 8 times more common among men and usually occurs between 20 and 50 years of age [2]. An incidence of $0.01 \%$ has been reported in the general population and $0.008 \%$ per year during pregnancy [4] [5].

CSCR is responsible for a macular syndrome, usually unilateral, with decreased visual acuity and metamorphopsia.

Clinically the eye fundus of our patient showed an isolated macular bubble without exudate or hemorrhage.

White retinal exudates are found in CSCR and pregnancy cases, in contrast to cases not associated with pregnancy [6].

Sunness et al. [6] reported retinal exudates associated with CSCR during pregnancy in 3 of 4 of their patients, all Caucasians; such precipitates were reported to be independent of race. Mayo and Tolentino [7] found these exudates in a 32-year-old pregnant black woman with CSCR.

Although the presence of retinal exudate was common in CSCRs on pregnancy (50\% - 90\%), Quillen et al. [8] reported that the occurrence of such exudates is more common in patients who were on corticosteroids. The absence of retinal exudate in our patient could be explained by the absence of corticosteroid use before or during pregnancy. The underlying mechanism of CSCR during pregnancy remains unclear. It is believed that high levels of endogenous steroid cortisol [9] could trigger a chain of events altering the blood-retinal barrier, choriocapillaris and pigment epithelium, resulting in focal areas of increased permeability, and consequently precipitating CSCR.

Optical coherence tomography (OCT) showed a central macular thickness of 894 microns with pigment epithelial detachment representing the leakage zone 
(Figure 1).

OCT and clinical examination were suggestive of central serous chorioretinopathy.

We did not perform fluorescein retinal angiography as a precautionary measure, although it is not an absolute contraindication during pregnancy.

The conditions to be considered in the differential diagnosis would include exudative retinal detachment from preeclampsia, but this is quite unlikely because of the absence of hypertension, proteinuria, and pedal edema in our patient.

The course of CSCR in our patient was self-limiting, and she did not require treatment with ophthalmological surveillance. Treatment, however, is occasionally necessary in patients with CSCR. Indications for treatment include persistence of a serous retinal detachment for more than four months, recurrence in an eye with visual deficit from previous CSCR, presence of visual deficits in opposite eye from previous episodes of CSCR, and occupational or other patient needs the necessitate prompt recovery of vision. Retinal laser therapy has been found to be quite effective in CSCR and is the current standard of care.

Indeed, different treatment modalities have been proposed for the treatment of CSCR, the vast majority of these cases disappear spontaneously over time, ranging from six weeks to one year [10].

Photodynamic therapy with visudyne and laser photocoagulation of the visible leak point(s) in angiography if these leak points are outside the visual axis remain controversial; however, this may be justified in chronic CSCRs where symptoms persist for months or years and an intervention may be justified.

Corticosteroids should be stopped whenever possible. The role of anti-VEGF (Vascular Endothelial Growth Factor) is not yet established in the treatment of CSCR [11] [12]. Medical treatment with Eplerenone or spironolactone is being assessed.

The outcome was satisfactory marked by an uncorrected visual acuity of 1.0 and the control OCT returned strictly normal (Figure 2).

CSCR is more frequent in the $3^{\text {rd }}$ trimester. It can also occur in the $1^{\text {st }}$ or $2^{\text {nd }}$ trimester. Most CSCRs spontaneously heal in 4 - 8 weeks postpartum, with visual acuity recovery in 3 to 6 months. But alterations of pigment epithelium, metamorphopsia, and central visual field involvement can rarely persist with severe visual loss reported in $5 \%$ of CSCRs.

Recurrence during subsequent pregnancies is possible. Mujaini observed alternating central serous chorioretinopathy in two consecutive pregnancies in a 31-year-old woman. The first episode was in the left eye and the second in the right eye [13].

Recurrence during future pregnancies are known in the literature and in 10\% of cases could have three recurrences or more [14].

Half of the recurrences occur in the first year but can occur up to 10 years after the first episode [14]. 


\section{Conclusion}

This case report reaffirms the importance of ophtalmology examination in the case of lossed visual acuity in pregnancy. Hence early and correct diagnosis of this condition may avert superfluous diagnostic and therapeutic measures in pregnancy.

\section{Consent}

Written informed consent was obtained from the patient for publication of this Case report and any accompanying images.

\section{Conflicts of Interest}

There are no conflicts of interest.

\section{References}

[1] Gass, J.D.M. (1987) Idiopathic Central Serous Chorioretinopathy. In: Stereocopic Atlas of Macula Diseases. Diagnosis and Treatment, 3rd Edition, C.V. Mosby Company, St Louis, MO, 46-59.

[2] Bouzas, E.A., Karadimas, P. and Pournaras, C.J. (2002) Central Serous Choroidoretinopathy and Glucocorticoids. Survey of Ophthalmology, 47, 431-448. https://doi.org/10.1016/S0039-6257(02)00338-7

[3] Marmor, M.F. (1988) New Hypothesis on the Pathogenesis and Treatment of Serous Retinal Detachment. Graefe's Archive for Clinical and Experimental Ophthalmology, 226, 548-552. https://doi.org/10.1007/BF02169203

[4] Liew, G., Quin, G., Gillies, M. and Fraser-Bell, S. (2013) Central Serous Chorioretinopathy: A Review of Epidemiology and Pathophysiology. Clinical \& Experimental Ophthalmology, 41, 201-214. https://doi.org/10.1111/j.1442-9071.2012.02848.x

[5] Said-Ahmed, K., Moustafa, G. and Fawzy, M. (2012) Incidence and Natural Course of Symptomatic Central Serous Chorioretinopathy in Pregnant Women in a Maternity Hospital in Kuwait. Middle East African Journal of Ophthalmology, 19, 273-276. https://doi.org/10.4103/0974-9233.97920

[6] Sunness, J.S., Haller, J.A. and Fine, S.L. (1993) Central Serous Chorioretinopathy and Pregnancy. Archives of Ophthalmology, 111, 360-364.

https://doi.org/10.1001/archopht.1993.01090030078043

[7] Mayo, G.L. and Tolentino, M.J. (2005) Central Serous Chorioretinopathy in Pregnancy. The New England Journal of Medicine, 353, e6. https://doi.org/10.1056/NEJMicm040678

[8] Quillen, D.A., Gass, D.M., Brod, R.D., Gardner, T.W., Blankenship, G.W. and Gottleib, J.L. (1996) Central Serous Choroidopathy in Women. Ophthalmology, 103, 72-79. https://doi.org/10.1016/S0161-6420(96)30730-6

[9] Bouzas, E.A., Scott, M.H., Mastorakos, G., Chrousos, G.P. and KaiserKupfer, M.I. (1993) Central Serous Chorioretinopathy in Endogenous Hypercortisolism. Archives of Ophthalmology, 111, 1229-1233. https://doi.org/10.1001/archopht.1993.01090090081024

[10] Sunness, J.S. (1992) Pregnancy and the Eye. Ophthalmology Clinics of North America, 5, 623-640.

[11] Mak, S.T. and Wong, A.C. (2013) Single-Session Combined Photodynamic Therapy 
with Verteporfin and Intravitreal Anti-Vascular Endothelial Growth Factor Therapy for Chronic Central Serous Chorioretinopathy: A Pilot Study at 12-Month Follow-Up. Graefe's Archive for Clinical and Experimental Ophthalmology, 251, 401-402. https://doi.org/10.1007/s00417-011-1883-6

[12] Arevalo, J.F. and Espinoza, J.V. (2013) Combined Photodynamic Therapy with Verteporfin and Intravitreal Anti-Vascular Endothelial Growth Factor Therapy for Chronic Central Serous Chorioretinopathy. Graefe's Archive for Clinical and Experimental Ophthalmology, 251, 403-404. https://doi.org/10.1007/s00417-011-1884-5

[13] Al-Mujaini, A. and Wali, U. (2014) Alternating Central Serous Chorioretinopathy in Two Consecutive Pregnancies. Oman Journal of Ophthalmology, 7, 87-89. https://doi.org/10.4103/0974-620X.137169

[14] Hussain, D. and Gass, J.D. (1998) Idiopathic Central Serous Chorioretinopathy. Current Ophthalmology, 46, 131-137.

\section{Abbreviations \\ OCT: Optical Coherence Tomography \\ CRSC: Central Serous Chorioretinopathy \\ VEGF: Vascular Endothelial Growth Factor \\ EPR: Retinal Pigment Epithelium}

